\section{Nevirapine nanosuspension: comparative investigation of production methods}

\author{
Ranjita Shegokar, ${ }^{1,2}$ Kamalinder K. Singh, ${ }^{1}$ \\ Rainer H. Müller ${ }^{2}$ \\ 'C.U. Shah College of Pharmacy, S.N.D.T \\ Women's University, Mumbai, India; \\ ${ }^{2}$ Freie Universität Berlin, Institute of \\ Pharmacy, Department of \\ Pharmaceutics, Biopharmaceutics \& \\ NutriCosmetics, Berlin, Germany
}

\section{Abstract}

Increasing number of antiretroviral drugs coming from high throughput screening besides their high dose has poor solubility profile. Formulation development of these drugs is a major obstacle to their clinical application. To overcome extremely low water solubility and associated poor bioavailability they can be formulated as nanosuspensions. This paper is not only focuses on production of parenteral nevirapine nanosuspensions but also on scaling up of formulations for clinical use. Lab scale (APV LAB 40, $40 \mathrm{~mL}$ ) and medium scale (Avestin C50, $2 \mathrm{~kg}$ ) production was performed using piston gap high pressure homogenization ( $\mathrm{HPH})$, while the feasibility for pilot scale up was checked using a bead milling technique in continuous mode (PM, Bühler PML-2). Nanosuspension was characterized for particle sizes, zeta potential, crystallanity and stability. The mean particle sizes for lab scale, medium scale and pilot scale production obtained were $481 \mathrm{~nm}, 429 \mathrm{~nm}$ and $211 \mathrm{~nm}$, respectively. Independent of the production method (lab and pilot scale) all processed formulations showed more or less similar zeta potential $(\sim 15 \mathrm{mV})$ in conductivity adjusted water. Long term stability over 1 year showed significant increase in particle size at all storage conditions for lab scale and medium scale production (high energy size reduction) whereas they remained physically stable (with negligible increase) for the milled product (low energy size reduction). As the technology has been scaled up successfully for nevirapine nanosuspension, the product can be considered for commercial exploitation. The prepared nevirapine nanosuspensions can be administered for parenteral or oral use.

\section{Introduction}

After successful development of pharmaceutical formulation at lab scale with promising in vivo results, it is an important step to make clinical batch towards first human trials. A successful introduction of a drug delivery system to the market mainly depends upon efficiency of equipment to produce large-scale batch (GMP production) at adequate manufacturing and labor costs. In addition, the production technology should allow ease of scale up from lab to industrial scale. ${ }^{1,2}$ Normally, from moving up in batch size by a factor of 10 to 100 various problems occur in obtaining a product of the same specifications. ${ }^{3}$ Many successful formulations at lab scale might fail during scaling up trials. Scaling up is a major obstacle especially for the introduction of particulate or colloidal drug carriers into the pharmaceutical market. Although large-scale production methods are established for liposomes, nanoemulsions and some microparticles still there are major problems in the establishment of large-scale production methods for nanosuspension and polymeric nanoparticles. An aseptic production makes final sterilization mandatory for parenteral product and it is considered to be one of the major hurdles preventing the successful introduction of the nanoparticles to the pharmaceutical market. Literature reports use of high pressure homogenization (HPH) and combination technology for the production of flavonoids nanocrystals e.g. apigenin, ${ }^{4}$ quercetin, ${ }^{5}$ rutin etc.

More than 20 years of intensive research has been invested in nanoparticle technology, ${ }^{6,7}$ but the output in terms of pharmaceutical products for the patients in comparison with cosmeceutical is rather low. Two major reasons for the lack of large-scale production methods for nanoparticles are that they include various technological problems (e.g., temperature control, pressure variation, sterilization issues, remains of toxic residues from the production process, stability issues, erosion of equipment surfaces etc.) and regulatory aspects such as suitability of the production unit and production process to be qualified and validated. A GMP production unit to produce nanoparticle batches of 2 to $10 \mathrm{~kg}$ is available with company such as Pharmtec (Milan, Italy) and SkyePharma (Muttenz/Basel, Switzerland), Dr. Rimpler GmbH (Hannover, Germany) and CLR GmbH (Berlin, Germany). The therapeutic strategy for the treatment of AIDS is very complex, long dosing regimens, costs, side effects, limitations of drug biodistribution, and variable pharmacokinetic patterns due to poor solubility. ${ }^{8}$ The solubility problem and dose related issue can be solved to some extent by converting high dose poorly soluble antiretrovirals drugs into nanocrystals. Nanocrystals are submicron colloidal dispersions of discrete particles in presence of surfactants, polymers or a mixture of both. The nanosuspensions can be used to formulate compounds that are insoluble in water, oils and organic media. ${ }^{9}$ Nanocrystals offer number of advantages over
Correspondence: Rainer H. Müller, Freie Universität Berlin, Institute of Pharmacy, Department of Pharmaceutics, Biopharmaceutics \& NutriCosmetics, Kelchstrasse 31, 12169 Berlin, Germany.

Tel. +49.30.838.506.96 - Fax: +49.30 .838 .506 .16 . E-mail: nanoteam@gmx.com

Key words: nevirapine, poor solubility, nanonization, nanosuspension, scale up.

Acknowledgement: the authors would like to thank Deutscher Akademischer Austausch Dienst for financial support.

Received for publication: 30 June 2011. Accepted for publication: 29 September 2011.

This work is licensed under a Creative Commons Attribution NonCommercial 3.0 License (CC BYNC 3.0).

\section{(O) Copyright R. Shegokar et al., 2011}

Licensee PAGEPress, Italy

Nanotechnology Development 2011; 1:e4 doi:10.4081/nd.2011.e4

other nanoparticles like, increased dissolution velocity (saturation solubility), enhanced adhesiveness properties, enhanced pharmacokinetic/biodistribution property, ${ }^{10}$ feasibility of surface modification, organ and/or cellular targeting, and ease of formulation. Nanosuspensions can be produced using two types of technique (i) top-down processes (nanonization) and (ii) bottom-up processes (controlled precipitation/ crystallization). ${ }^{11-13}$ Top-down technology has wide variety of advantages over bottom-up techniques. Among the top down techniques, HPH is a widely accepted method for production of nanosuspension, have been used for many years. ${ }^{9}$ In typical HPH, formed shear forces, elongation, turbulence and cavitation may lead to break-up of the droplets/particles through a sudden pressure drop of several hundred bars. ${ }^{14}$

Antiretroviral loviride nanosuspensions were prepared by milling $(264 \pm 14 \mathrm{~nm})$ at lab scale and freeze-dried using sucrose as cryoprotectant to obtain nanopowder (560-590 $\mathrm{nm}) .{ }^{15}$ Loviride nanosuspension showed higher dissolution profiles compared to that of untreated drug powder after milling. In transepithelial transport experiment conducted on Caco-2 cell, nanopowder showed a significantly superior cumulative loviride amount transported to cells a $(1.59 \pm 0.02 \mu \mathrm{g})$ as compared to the physical mixture (sucrose and untreated loviride, $0.93 \pm 0.01 \mu \mathrm{g}$ ) and the untreated loviride $(0.74 \pm 0.03 \mu \mathrm{g})$ at $120 \mathrm{~min} .{ }^{15}$ Nanonization approach was not only tried for existed drug but also a new chemical entity like BMS-488043 to improve suboptimal bioavailability by using nanonization (media milling, polystyrene beads $500 \mu \mathrm{m})$. 
Amorphous intermediates of BMS-488043 were prepared using spray drying and flash evaporation. In vivo pharmacokinetic studies on tablet (micronized BMS-488043) and capsules (containing amorphous drug/ nanosuspension) conducted in beagle dogs showed significant increase in Cmax (4.7 fold) as well $\mathrm{AUC}_{0}{ }^{24}$ (4.6 fold) by nanonization in fasted state. The $\mathrm{AUC}_{0}{ }^{24}$ of nanonized drug was comparatively lower than that of amorphous coprecipitates. All above studies indicate successful use of nanosuspension for HIV chemotherapy. In our previous study, nevirapine nanosuspension showed potential for HIV cellular and anatomical (organ specific targeting) reservoir targeting when tested in vitro ${ }^{16}$ and in vivo. ${ }^{17}$ Various physical and chemical stability studies supported the consideration of this formulation for further development. Our developed nevirapine nanosuspension formulation is promising candidate for clinical development. Therefore, in this study we checked the feasibility of large-scale production from lab scale ( $40 \mathrm{~g}$ batch) to ( $>2 \mathrm{~kg}$ batch). The data obtained with continuous mode arrangement of bead mill is transferable to that of discontinuous mode up to few hundred-kilogram batch sizes. Nanosuspensions obtained were characterized for particle size, zeta potential and stability. Finally, HPH production method was compared with milling technique in terms of effect on physicochemical characteristic of final formulation. All productions carried out were non sterile, however sterile production is possible using Avestin C50 and LAB 40.

\section{Materials and Methods}

\section{Materials}

Nevirapine (nevirapine, Alkem Laboratories, India), stabilizers like, Poloxamer 188 (BASF $\mathrm{GmbH}$ ), Tween 80 (Uniquema, Belgium), VolpoL4 (Croda $\mathrm{GmbH}$ ), Plasdone (International Specialty Products, India) and polyvinyl pyrrolidone K 25 (PVP, Signet Chemical Corporation, India) were received as gift samples. Sodium chloride ( $\mathrm{NaCl} 0.9 \%)$ solution was obtained from B. Braun Melsungen AG, Germany.

\section{Preparation of nanosuspension}

Nanosuspensions were prepared by dispersing coarse nevirapine $2.0 \%(\mathrm{w} / \mathrm{w})$ powder in $2.8 \%(\mathrm{w} / \mathrm{w})$ aqueous surfactant solution containing $1 \%$ Tween $80,0.9 \%$ VolpoL4, 0.1\% Plasdone, $0.5 \%$ Poloxamer and $0.3 \%$ PVP. Premixing was performed with a high shear Remi overhead stirrer followed by Ultra Turrax equipped with rotor T25 (Janke \& Kunkel, Staufen, Germany) for $1 \mathrm{~min}$ at $9500 \mathrm{rpm}$. A prepared coarse suspension was then processed using high-pressure homogenizer or bead milling.

\section{Lab scale production using APV LAB 40}

Laboratory scale production of nanosuspension can be performed using a piston-gap homogenizer (Micron LAB 40, APV Homogenizer GmbH, Germany)..$^{18}$ It allows production of $20-40 \mathrm{~mL}$ product at various pressures ranging 100 to 1500 bar. The aqueous dispersion is pressed by a piston through a small homogenization gap of approx. $3 \mu \mathrm{m}$ (at a pressure of 1500 bar). ${ }^{1,19}$ The production process is discontinuous with LAB 40, the system needs to be dismantled before each homogenization cycle which makes production time consuming. APV LAB40 has biggest advantage of extremely low sample volume, which is very important for expensive or for limited availability compound (e.g. new chemical entity, NCE). Figure 1 shows the principle mode of operation of the LAB 40 unit. Two step procedure is generally applied for production of nanosuspension firstly premilling, which is carried out at lower pressure like 200-1000 bar for two cycles) to break large crystals which further avoids blocking of homogenization gap. Second step involves actual diminution at higher pressure (1300-2000 bar). Generally, 20-30 passes are required to obtained nanonized product depending upon the physical characteristic of starting material.

Nevirapine coarse suspension $(40 \mathrm{~mL})$ was subjected to pre-milling (200, 500 and 1000 bar 2 cycles each) and actual homogenization at 1500 bar for 20 cycles. Temperature of homogenization tower was maintained at $4{ }^{\circ} \mathrm{C}$ while nanosuspension sample was cooled after each 5 cycles. Sampling was performed after premilling and 1, 5, 10, 15 and 20 homogenization cycles.

\section{Medium scale production using Avestin C50}

EmulsiFlex-C50 (Avestin C50, Avestin Inc. Ottawa, Canada) can be employed for production ranging from 15 to $50 \mathrm{~L} / \mathrm{h}$ having hold back sample volume of $4 \mathrm{~mL}$. It is widely used for cell rupture and production of liposomes, nanoemulsion and dispersions. It allows production at pressure range of 300 to 2000 bar. It is approved homogenizer by official authorities like Food and Drug Administration and it allows sterilization in place. Another advantage include that it allows production at varied pressure and sizes. Avestin C50 can be operated in both discontinuous and continuous mode for production of nanocarriers (Figure 2). The reduction in particle size depends upon the hardness of drug, homogenization time and pressure applied.

Nevirapine coarse suspension $(2 \mathrm{~kg}$ ) was homogenized using EmulsiFlex C50 for 30 min in continuous mode. The homogenizer was pre cooled by passing ice cold distilled water at $4^{\circ}$ $\mathrm{C}$ while during homogenization temperature of nanosuspension was controlled using water bath. Sampling was performed after 5, 10, 15, 20, 25 and $30 \mathrm{~min}$.

\section{Bead milling}

Bead milling is another technique used to prepare nanosuspensions..$^{20,21}$ NanoCrystal is a patent protected technology developed by Liversidge et al. ${ }^{22}$ In this technique, the drug nanoparticles are obtained by subjecting the drug to media milling (e.g. water, stabilizer solution or buffer). High energy and shear forces generated as a result of impaction of the milling media or beads with the drug providing necessary energy input to disintegrate the microparticle drug into nanonized particles. This technique provides advantage of producing very viscous suspensions with $20-30 \%$ higher solid content that cannot be produced with HPH, which can be further diluted with stabilizer solution to produce desired concentration of nanosuspension (Figure 3). The major concern with this method is the residues of milling media remaining in the

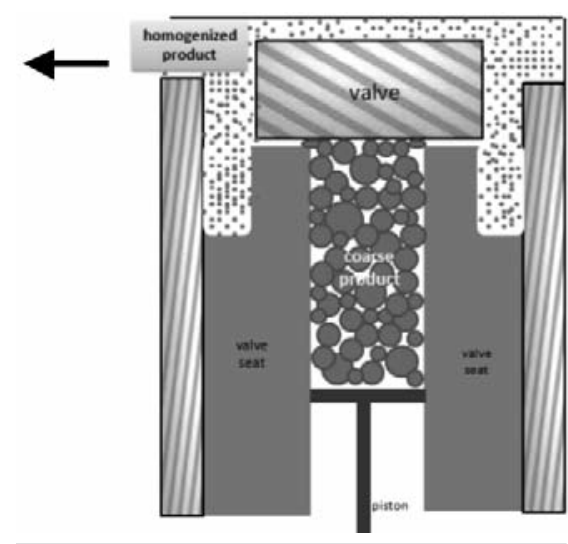

Figure 1. Production mode of discontinuous APV Micron LAB40, where nanosuspension pressed down through narrow homogenization gap under pressure.

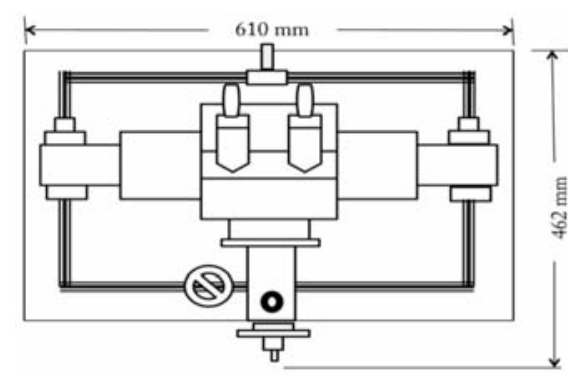

Figure 2. Production mode of Avestin C50, in continuous mode nanosuspension was circulated through homogenizer under high pressure for $30 \mathrm{~min}$. (modified after http://www.avestin.com). 
finished product could be problematic for administration. ${ }^{23}$ This technology was extended further by Müller group where nanosuspension prepared by combination technology (CT process, milling followed by HPH) to get more stable nanosuspension (Figure 3). Aqueous nevirapine coarse suspension $(120 \mathrm{~mL})$ was milled using Bühler PML-2 bead mill (Bühler $\mathrm{AG}$, Switzerland) for $3 \mathrm{~h}$ in continuous mode using 0.2-0.4 mm yttria stabilized zirconium oxide beads at $1000 \mathrm{rpm}$ at $4^{\circ} \mathrm{C}$. Sampling was performed after 5, 10, 15, 20, 25 and 30 minutes. All collected samples were subjected for size analysis.

\section{Characterization of nanosuspensions}

Prepared nanosuspensions were evaluated for color, $\mathrm{pH}$ and redispersibility. The obtained nevirapine nanosuspensions were characterized for mean particle size using a photon correlation spectroscopy (PCS) and laser diffractometry (LD). Zeta potential was determined in water (50 $\mu \mathrm{S} / \mathrm{cm}$ ) and original surfactant solution. Light microscopy was performed to access the presence of possible aggregates.

\section{Particle size determination}

\section{Photon correlation spectroscopy}

Photon correlation spectroscopy (PCS) measurements were performed using a Zetasizer Nano ZS (Malvern Instruments, UK). The z-average (intensity weighted mean diameter of the bulk population) and the polydispersity index (PI, measure for the width of the size distribution) of the sample were recorded at $25^{\circ} \mathrm{C}$. PCS measurements were performed on diluted nanosuspension in distilled water. Total ten measurements were performed and the mean value is reported.

\section{Laser diffraction}

Laser diffraction (LD) particle analysis was performed using a Mastersizer 2000 equipped with Hydro S sample dispersion unit (Malvern Instruments, UK) in deionized water. The $\mathrm{LD}$ volume weighted diameters were used as characterization parameters using real refractive index (1.56) and imaginary refractive index $(0.01)$. The LD95\% and LD99\% are sensitive parameters to quantify presence of potential large sized particles, e.g. larger crystals that may remain in the suspension during homogenization or aggregates formed due to insufficient stabilization.

\section{Light microscopy}

Light microscopy (Ortophlan, Germany) was performed to analyze the morphology ${ }^{19}$ of the particles and to observe the presence or absence of larger crystals or aggregates using 600 fold magnification. Photomicrographs were taken for each sample.

\section{Zeta potential determination}

Zeta potential (ZP) measurements were performed using a Malvern Zetasizer Nano ZS
(Malvern Instruments, UK). The Zetasizer Nano measures the electrophoretic mobility of the particles and converts it into zeta potential. The surface charge of particles mainly depends on the dispersion medium used therefore, zeta potential was determined in Milli- $Q$ water (conductivity adjusted to $50 \mu \mathrm{S} / \mathrm{cm}$ using $0.9 \% \mathrm{NaCl}$ solution) and in the original surfactant solution.

\section{X-Ray diffractometry}

$\mathrm{X}$-Ray diffractometry (XRD) was performed at room temperature with a Philips X-ray Generator PW 1830 (Philips, Netherlands) for bulk and prepared nanosuspensions to assess the degree of crystallanity. The diffraction pattern was measured at a voltage of $40 \mathrm{kV}$ and a current of $40 \mathrm{~mA}$ in the $2 \theta$-region of $4^{\circ}-60^{\circ}$ coupled with a model PW18120 Goniometer detector. Powdered drug or air dried nanosuspension was directly mounted on to small fiber plate. All samples were rotated during measurement at 1 rotation/s to allow reproducibility of measurements.

\section{Long term stability}

Long term stability for 12 months was performed on all produced nanosuspensions (HPH, bead mill processed) formulations. The nanosuspensions were stored at refrigeration (RF, $4^{\circ} \mathrm{C}$ ), room temperature (RT) and $40^{\circ} \mathrm{C}$ degree temperature. Samples were analyzed for particle sizes as function of time on the day of production, 1, 3, 6 and $12 \mathrm{month} / \mathrm{s}$ (data reported in graph is for 1, 6 and 12 month/s).

\section{Results and Discussion}

\section{Feasibility of production of nanosus- pensions}

Nevirapine is an antiretroviral drug, which has low water solubility (pKa of 2.8) and high intestinal permeability (BCS class II). Generally, in commercial products it is used along with other antiretroviral drugs like stavudine, lamivudine etc. in fixed dose. It is available as tablets (anhydrous form, fine grains) and as suspensions (hemihydrates form, prismatic crystals). Although, after oral administration the drug get absorbed rapidly, however at higher doses $<50-60 \mathrm{mg}$ nevirapine exhibit distinct rate limited absorption due to solubility issues. ${ }^{24,25}$ Based on available information about nevirapine, alternative formulation approach would be helpful to solve solubility issue i.e. nanonization. As particle size is determining parameter in the dissolution rate enhancement. Nanonization provides increased surface area for dissolution thereby enhancing the intrinsic solubility ${ }^{26}$ of drug. This study demonstrates feasibility of lab to industrial scale of production of nevirapine nanosuspension using high pressure homogenization (LAB 40 and Avestin C50) and milling technique. White colored homogeneous nanosuspension showed excellent redispersibility within two strokes and had pH between 6.87-7.02. The reason for selecting lower concentration $2 \% \mathrm{w} / \mathrm{w}$ for production of parenteral nanosuspension was to achieve maximum size reduction however for intravenous administration preferably size below $5 \mu \mathrm{m}$ is recommended to avoid capillary blockade. However concentrated nanosuspensions (up to $20 \%$ solid content) can be processed using $\mathrm{HPH}$ and milling.

\section{Laboratory scale production}

Piston-gap homogenizers are typically used for the production of nanocrystals. Homogenization of nanosuspension was performed at room temperature using a Micron LAB 40 (discontinuous version). As a pre-milling step, a pressure profile was run, i.e. 2 cycles at 200,500 bar and at 1000 bar. Actual homogenization was performed at increased pressure of 1500 bar for 20 cycles. The higher homogenization pressure lead to distinctly smaller particle size and lower content of micrometer particles. ${ }^{27}$ It confirmed the general rule that higher pressures lead to finer particles. The particle size of suspension became smaller with increasing homogenization pressure, which is in agreement with the homogenization theory. A higher homogenization pressure leads to a higher powder density and subsequently to an increase of the dispersitivity index. It should be noted that too high homogenization pressure can lead to particle aggregation with increasing cycle numbers. ${ }^{28}$

Figure 4 shows the mean PCS diameter of bulk population and LD as a function of the number of homogenization cycles. A decrease in mean particle size with increase in number

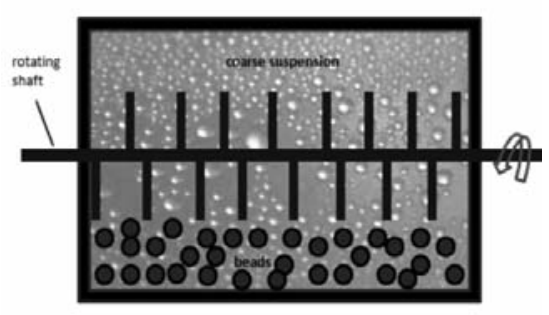

Figure 3. Cross section of bead mill loaded with beads which are available in varying sizes from 0.1 to $0.8 \mathrm{~mm}$.

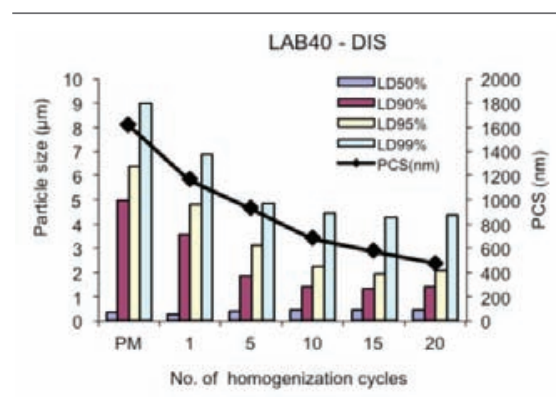

Figure 4. Effect of premilling (PM) and number of homogenization cycles on mean particle size (PCS) and particle size distribution (LD50\%, LD90\%, LD95\%, LD99\%) using LAB40 in discontinuous (LAB40 DIS) mode on the day of production. 
of homogenization cycles was observed till fifteen cycles $(560 \pm 12 \mathrm{~nm}$ and PI of $0.226 \pm$ 0.097). Further increase in number of cycles did not show any difference in particle size reduction. Twenty homogenization cycles were sufficient to produce a white colored homogeneous nanosuspension with a mean diameter of $481 \pm 10 \mathrm{~nm}$; with PI of about $0.212 \pm 0.085$. Further decrease in particle size was not observed when increasing the cycle number ( $>20$ cycles, data not reported), indicating that the limiting dispersivity at a given power density for this drug has been reached. LD measurements reflected that continuous reduction in the diameter $90 \%$ and $99 \%$ can be obtained till 15th homogenization cycle but after that the diameter remained practically unchanged till 20th homogenization cycle.

\section{Medium scale batch production}

Nanosuspensions are a highly dispersed system; Avestin C50 is widely used in production of such dispersed systems. Efficacy of Avestin C50 to produce nanosuspension was compared at same pressures as that of piston gap homogenizer i.e. 1500 bar. A decease in mean particle size was observed up to $30 \mathrm{~min}$ (Figure 5) Particle size was reduced from $20 \mu \mathrm{m}$ of coarse powder to $9621 \pm 30 \mathrm{~nm}$ after $5 \mathrm{~min}$ homogenization which was further decreased to $429 \pm 16$ after $30 \mathrm{~min}$ at $1500 \mathrm{bar}$ (without any premilling step). Maximum dispersivity ( $\mathrm{PI}=0.158)$ was obtained after 20 min homogenization further increase in homogenization time resulted in slight decrease in particle size but no major change were observed in dispersivity after that. The LD diameter LD90\% and 99\% was $1.235 \mu \mathrm{m}$ and $4.120 \mu \mathrm{m}$, respectively, almost below $5 \mu \mathrm{m}$ making it suitable for intravenous administration. Even though the nanosuspensions produced with Avestin C50 and LAB 40 has similar mean particle size, but broader particle size distribution can be seen for formulation produced using Avestin C50. It might be due to batch volume that could have affected the dispersivity i.e. homogeneity of nanosuspension. In small batch size production, it is easy to control production parameters to get desired particle size. However, the same parameters cannot be applicable for clinical batch production, during scaling-up much attention should provided to the determining parameters like batch volume, homogenization time, number of homogenization cycles and equipment limitations. ${ }^{2}$ In previous experiments done in another laboratory using Avestin C50, suspension was circulated at various pressures (1000-1500 bar) to get almost similar results for PCS $(457 \mathrm{~nm}, \mathrm{PI}=0.578)$, but for $\mathrm{LD} 95 \%$ the values was around $2200 \mathrm{~nm}$ and for $\mathrm{LD} 95 \%$ around $2500 \mathrm{~nm}$.

\section{Viability of large-scale production}

Bead milling is very effective method used in production of nanosuspension. ${ }^{15,27}$ Viability of large-scale production was checked using small milling chamber (150 $\mathrm{mL}$ in volume). Reduction in particle size was observed which in good agreement with previous experience (Figure 6). Particle size was drastically decreased with milling up to $15 \mathrm{~min}$, maximum dispersivity was obtained after 60 min milling. Further increase in milling time did not show any marked effect on particle size. PCS showed mean particle size of $211 \pm 10 \mathrm{~nm}$ with polydispersity index of $0.157 \pm 0.087$ after $30 \mathrm{~min}$ milling which was remained almost constant for next $180 \mathrm{~min}$ (at $3 \mathrm{~h}$, the mean diameter obtained was $202 \pm 12 \mathrm{~nm}$ with PI of $0.182 \pm 0.093)$. The nanosuspensions produced with bead milling have excellent redispersibility. The PCS diameter of $637 \pm 64 \mathrm{~nm}$ was obtained only after one minute milling and was further reduced to $416 \pm 48 \mathrm{~nm}$ after $2 \mathrm{~min}$ (almost same size as obtained with LAB 40 and Avestin C50) with polydispersity index of $0.315 \pm 0.106$.

Laser diffractometry volume diameter i.e. LD99\% was observed to be $1.034 \mu \mathrm{m}$ after $3 \mathrm{~h}$ milling indicated that almost all particles were below one micron. Batch transfer from continuous production mode can easily transferred to discontinuous mode with change of few nanometers in particle size. Nanosuspension containing up to $20 \% \mathrm{w} / \mathrm{w}$ solid content can be easily milled in discontinuous mode for 6 to 7 cycles using bigger chamber size. Feasibility of bead milling for production of apigenin nanocrystals 0.5 to $3 \mathrm{~kg}$ batch was successfully achieved by Al Shaal et al. ${ }^{4}$

\section{Comparative production data}

In lab scale production using LAB 40, the mean particle size of $481 \mathrm{~nm}$ was obtained after 20 cycles with polydispersity index of 0.212 , while $429 \mathrm{~nm}$ ( $\mathrm{PI}=0.158$ ) for medium scale batch (2 kg) using Avestin C50. This particle size remained unchanged even applying more homogenization cycles or time. A parenteral nanosuspension of nevirapine can be easily produced, using LAB 40 (20 cycles) or Avestin C50 (30 min). Figure 7 shows comparative particle size data of LAB 40, Avestin C50 and bead milling produced batches and their storage stability. LD volume diameters $50 \%$ was $0.448 \mu \mathrm{m}, 0.328 \mu \mathrm{m}$ and LD90\% was 1.429 $\mu \mathrm{m}$ and 1.235 for LAB 40 and Avestin C50 processed batches, respectively (Figure 7). Minor differences $(\sim 5-8 \mathrm{~nm})$ in mean particle sizes or polydispersity indices do not hinder the performance of formulation in vivo. The increase in particle size or possible aggregation during storage stability for Avestin C50 produced nanosuspension could be due to heat transfer from metal surface during production of large size batch ( $40 \mathrm{~g}$ to $2 \mathrm{~kg}$, factor of 40 ). This increase in particle size during stability could be avoided by lyophilizing the nanosuspension immediately after production. For pilot scale, trial production using smaller size milling chamber showed interesting results, mean particle size noted was $202 \mathrm{~nm}$ (PI= 0.182 ) and LD95\% of $0.893 \mu \mathrm{m}$ indicating narrowing of the size distribution and breaking of large crystals efficiently. Based on particle size data there are clear differences between PCS and LD size values for nanosuspension produced using two techniques. HPH processed nanosuspensions are ideal for oral application while for parenteral application milled nanosuspension can be considered. The production method selected in this work gives reproducible results and is already being in

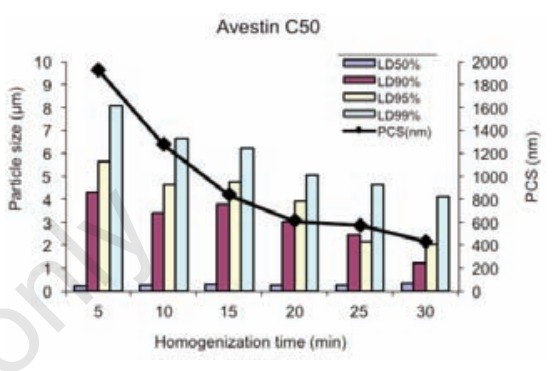

Figure 5. Effect of homogenization time on mean particle size (PCS) and particle size distribution (LD50\%, LD90\%, LD95\%, LD99\%) using Avestin C50 in continuous mode on the day of production.

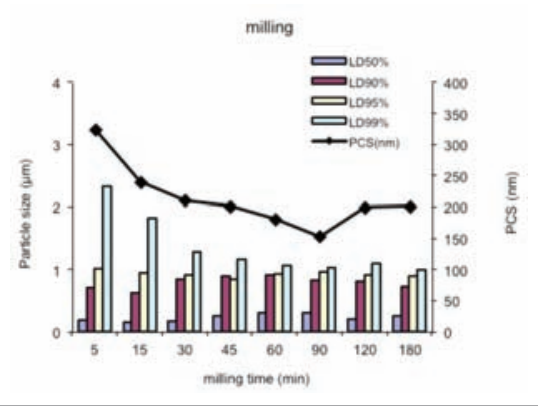

Figure 6. Reduction in mean particle size (PCS) and particle size distribution (LD50\%, LD90\%, LD95\%, LD99\%) as function of milling time on the day of production.

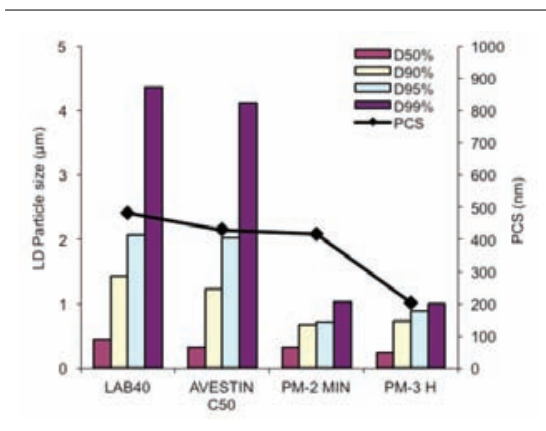

Figure 7. Efficacy of production techniques (high pressure homogenization LAB 40 and Avestin C50 and bead milling (PM)) and their effect on particle size reduction measured as mean diameter (PCS) and particle size distribution (LD50\%, LD90\%, LD95\%, LD99\%). 
industry for production of nanocrystals for dermal application. Highest diminution in particle size was obtained when milling was used. Lyophilization is highly recommended for $\mathrm{HPH}$ (LAB 40 and Avestin C50) processed nanosuspension.

\section{Zeta potential}

The zeta potential measurement indicates formation of stable system avoiding aggregation of fine particle. Zeta potential measurements were performed in $50 \mu \mathrm{S} / \mathrm{cm}$ water as it helps to reduced interday variability in measurements rather than pure water (with no conductivity adjustments). Surface charge measured in water $(50 \mu \mathrm{S} / \mathrm{cm})$ is very close to the Stern potential which is related to the potential of the particle surface (Nernst potential). All produced nanosuspensions exhibited negative zeta potential value around $-15 \mathrm{mV}$ (Table 1) which indicates a moderately charged negative surface and related stability. Higher is the zeta $(>+30 \mathrm{mV}$ or $>-30 \mathrm{mV})$ potential the higher is the stability. While drawing conclusion, one has to remember the rule of ZP values $\leq 30 \mathrm{mV}$ stability applies only to electrostatically stabilized colloidal systems. As nevirapine nanosuspension is prepared using combination of surfactants, which can provide the stability to nanosuspension by steric hindrance. A zeta potential measurement in original dispersion medium gives idea about the thickness of the diffuse layer. PVP and Poloxamer being a steric stabilizer used in formulation might have provided stability to the nano suspension despite lower zeta potential value.

\section{Light microscopy}

Light microscopy gives rough idea about particle size distribution and presence of possible aggregation. Figure 8 shows, formation of homogenous nanocrystals of nevirapine after production (left) and after one year of storage at room temperature (right). The presence of large crystals or small aggregates can be easily seen for stability sample under 600 magnifications using non-polarized light. The percentage of aggregation was much higher for HPH produced nanosuspension in comparison to milling technique. This is according to theory, milling being low energy process as compared to HPH. In milling, shearing of beads with drug crystals in production chamber, results in breaking down of particles. While in $\mathrm{HPH}$, a particle has to pass through a narrow gap of 3 $\mu \mathrm{m}$ at high pressure of 1500 bar. This might cause disruption of stabilizer layer around particle resulting in instability.

\section{X-ray diffraction}

Figure 9 shows $\mathrm{X}$ - ray diffraction patterns of nevirapine nanosuspension produced using various production techniques. The small halo region is due to amount of water present in air dried sample. The XRD patterns conforms the clear decrease in peak intensities for nanosuspension produced in given order $\mathrm{PM}>\mathrm{HPH}$ (LAB 40) > HPH (Avestin C50) without affecting its crystallanity). Differences in particle size for high pressure homogenized nanosuspensions using LAB 40 and Avestin C50 could be due to increased batch size and processing time. The high intensity peak of coarse nevirapine drug powder was found at $2 \theta$ of 9.1 , $14.3,18.26$ and 25.6 which markedly reduced after conversion into nanosuspension. Among the production techniques used, nanosuspensions processed using bead mill showed significant reduction peak intensities.

\section{Long term stability \\ Nanocrystals can be administered as an}
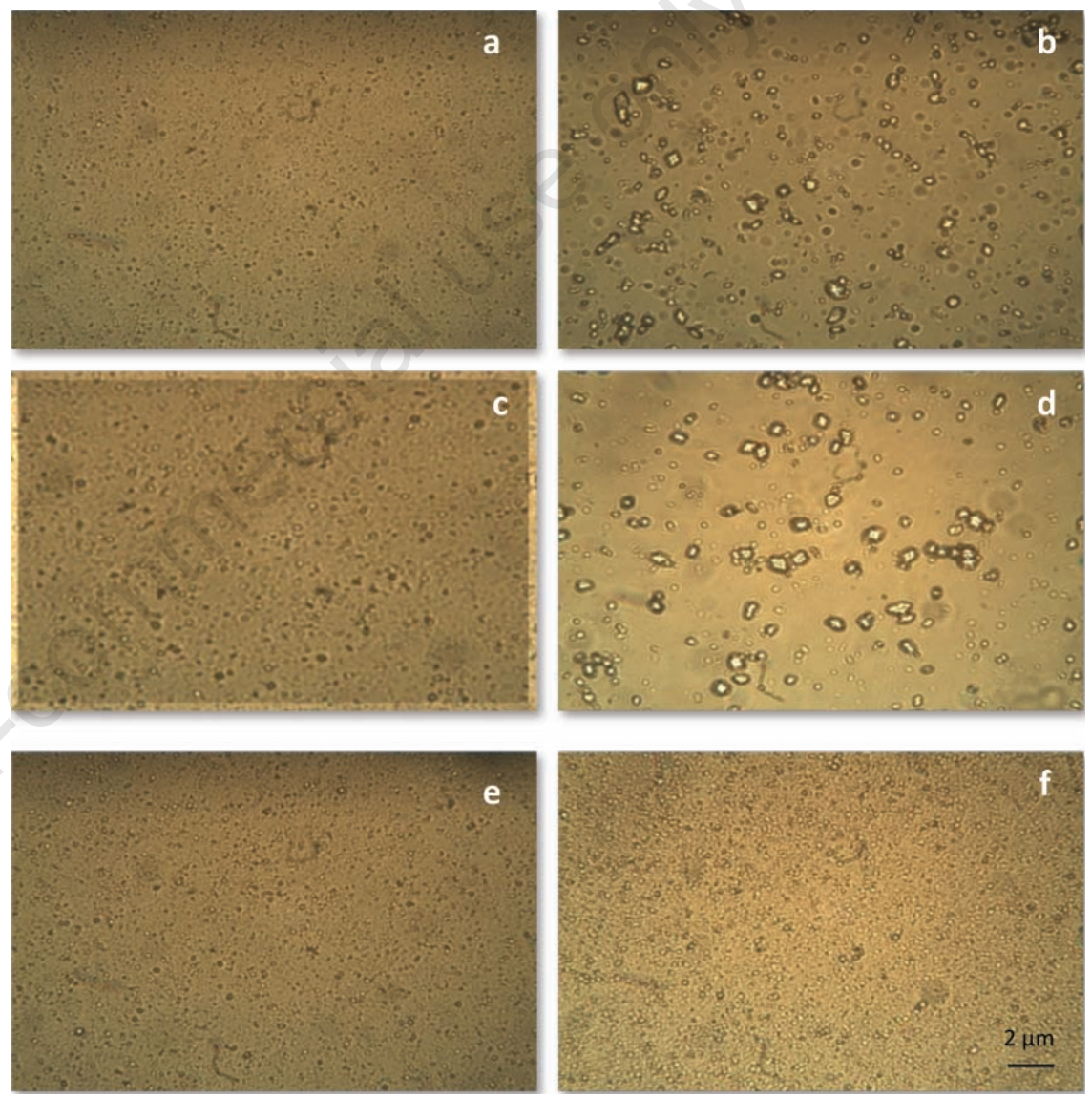

Figure 8. Photomicrographs of nevirapine nanosuspensions on day of production (left) and after one year of storage (right) LAB 40 (a, b), Avestin C50 (c, d) and bead milling (e, f) at room temperature under 600x magnification.

Table 1. Zeta potential of nevirapine nanosuspension on the day of production using various size reduction techniques.

\begin{tabular}{lcc} 
Production method & \multicolumn{2}{c}{$\begin{array}{c}\text { Zeta Potential (mV) } \\
\text { Original surfactant solution }\end{array}$} \\
APV LAB 40 (HPH) & $-15.2 \pm 1.12$ & $-10.3 \pm 1.45$ \\
Avestin C50 (HPH) & $-15.3 \pm 2.03$ & $-11.2 \pm 1.08$ \\
\hline Bühler PML-2 (milling) & $-16.8 \pm 1.87$ & $-10.7 \pm 1.64$ \\
\hline
\end{tabular}


year. Light microscopy confirmed the results obtained with LD and PCS showing presence of aggregates (Figure 10).

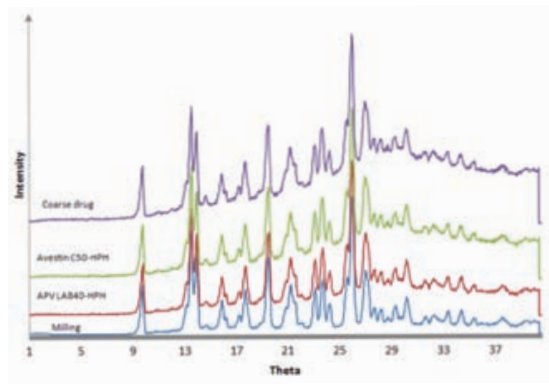

Figure 9. X-ray diffractograms of pure drug and nanosuspension prepared with HPH (APV LAB 40, Avestin C50) and bead milling techniques.
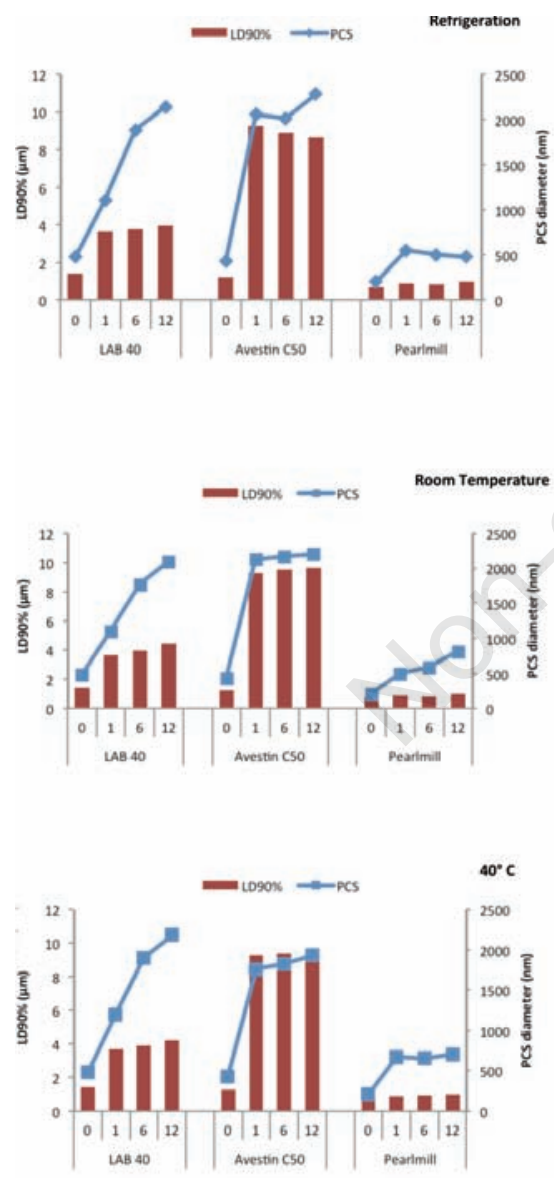

Figure 10. Long term stability $(0,1,6,12$ month) data of nevirapine nanosuspensions produced using high pressure homogenization and bead milling at refrigeration (above), room temperature (middle) and $40^{\circ} \mathrm{C}$ (below).

\section{Conclusions}

Thus, nevirapine nanosuspensions can be effectively scaled up using HPH and milling technique. In addition, nanosuspension with lower particle size distribution can be prepared using bead mill in concentrated form and can be further diluted to give desired dose. These concentrated nanosuspensions can also be converted to solid dosage form by means of spray drying, fluid bed drying or lyophilization. To prevent increase in particle size in case of parenteral application, it is advisable to lyophilize the nanosuspension immediately after production to maintain the integrity and performance of product.

\section{References}

1. Lai F, Sinico C, Ennas G, et al. Diclofenac nanosuspensions: influence of preparation procedure and crystal form on drug dissolution behaviour. Int $\mathrm{J}$ Pharm 2009;373:124-32.

2. Müller RH, Dingler A, Schneppe T, Gohla S. Large scale production of solid lipid nanoparticles (SLN ${ }^{\mathrm{TM}}$ ) and nanosuspensions (DissoCubes ${ }^{\mathrm{TM}}$ ). In: Wise D, editor. Handbook of Pharmaceutical Controlled Release Technology. New York: Marcel Dekker Inc; 2000. pp. 359-76.

3. Grau MJ, Kayser 0, Müller RH. Nanosuspensions of poorly soluble drugs-reproducibility of small scale production. Int J Pharm 2000;196:155-9.

4. Al Shaal L, Müller RH, Shegokar R. smartCrystal combination technology-scale up from lab to pilot scale and long term stability. Pharmazie 2010;65:877-84.

5. Kakran M, Sahoo NG, Shegokar R, et al. Fabrication of quercetin nanocrystals: Comparison of different methods. Eur $\mathrm{J}$ Pharm Biopharm 2011. [Epub ahead of print].

6. Mehnert W, Mäder K. Solid lipid nanoparticles: production, characterization and applications. Adv Drug Deliv Rev 2001;47:165-96.

7. Müller RH, Shegokar R, Keck CM. 20 years of lipid nanoparticles (SLN and NLC): present state of development and industrial applications. Curr Drug Discov Technol 2011;8:207-27.

8. Amiji MM, Vyas TK, Shah LK. Role of nanotechnology in HIV/AIDS treatment: potential to overcome the viral reservoir challenge. Discov Med 2006;6:157-62.

9. Keck CM, Müller RH. Drug nanocrystals of poorly soluble drugs produced by high pressure homogenisation. Eur J Pharm Biopharm 2006;62:3-16.
10. Kocbek P, Baumgartner S, Kristl J. Preparation and evaluation of nanosuspensions for enhancing the dissolution of poorly soluble drugs. Int $\mathrm{J}$ Pharm 2006;312:179-86.

11. Van Eerdenbrugh B, Van den Mooter G, Augustijns P. Top-down production of drug nanocrystals: nanosuspension stabilization, miniaturization and transformation into solid products. Int $\mathrm{J}$ Pharm 2008;364:64-75.

12. Patravale VB, Date AA, Kulkarni RM. Nanosuspensions: a promising drug delivery strategy. J Pharm Pharmacol 2004;56:827-40.

13. Shegokar R, Müller RH. Nanocrystals: Industrially feasible multifunctional formulation technology for poorly soluble actives. Int J Pharm 2010;399:129-39.

14. Krause KP, Müller RH. Production and characterisation of highly concentrated nanosuspensions by high pressure homogenisation. Int J Pharm 2001;214:214.

15. Van Eerdenbrugh B, Froyen L, Martens JA, et al. Characterization of physico-chemical properties and pharmaceutical performance of sucrose co-freeze-dried solid nanoparticulate powders of the anti-HIV agent loviride prepared by media milling. Int J Pharm 2007;338:198-206.

16. Shegokar R, Jansch M, Singh KK, Müller $\mathrm{RH}$. In-vitro protein adsorption studies on nevirapine nanosuspensions for HIV chemotherapy. Nanomedicine : nanotechnology, biology, and medicine 2011;7:33340.

17. Shegokar R, Singh KK. Surface modified nevirapine nanosuspensions for HIV viral reservoir targeting: In vitro and in vivo evaluation. Int J Pharm. 2011 Oct 1. [Epub ahead of print].

18. Liedtke S, Wissing S, Müller RH, Mäder K. Influence of high pressure homogenisation equipment on nanodispersions characteristics. Int J Pharm 2000 10;196:183-5.

19. Lindfors L, Skantze P, Skantze U, et al. Amorphous drug nanosuspensions. 1. Inhibition of Ostwald ripening. Langmuir 2006;22:906-10.

20. Shah T, Patel D, Hirani J, Amin AF. Nanosuspensions as a drug delivery systems: A comprehensive review. Drug Del Technol 2007;7:42-53.

21. Chingunpituk J. Nanosuspension Technology for Drug Delivery. Walailak J Sci Technol 2007;4:139-53.

22. Merisko-Liversidge EM, Liversidge GG. Drug nanoparticles: formulating poorly water-soluble compounds. Toxicol Pathol 2008;36:43-8.

23. Merisko-Liversidge E, Liversidge GG, Cooper ER. Nanosizing: a formulation approach for poorly-water-soluble com- 
pounds. Eur J Pharm Sci 2003;18:113-20.

24. Lamson MJ, Sabo JP, MacGregor TR, et al. Single dose pharmacokinetics and bioavailability of nevirapine in healthy volunteers. Biopharm Drug Dispos 1999;20:285-91.

25. Sarkar M, Perumal OP, Panchagnula R. Solid-state characterization of nevirapine. Indian J Pharm Sci 2008;70:619-30.
26. Müller RH, Shegokar R, Gohla S, Keck CM. Nanocrystals: Production, Cellular Drug Delivery, Current and Future Products- In Intracellular Delivery In: Ales P, editor. Fundamental Biomedical Technologies. New York: Springer; 2011. pp. 411-32.

27. Müller RH, Moschwitzer J, Bushrab FN. Manufacturing of nanoparticles by milling and homogenization techniques. In: Gupta
RB, Kompella UB, editors. Nanoparticle Technology for Drug Delivery (159 Aufl). New York: Taylor \& Francis Group; 2006. pp. 21-52.

28. Müller RH, Akkar A. Drug nanocrystals of poorly soluble drugs. In: Nalwa HS, editor. Encyclopedia of Nanoscience and Nanotechnology. Valencia, CA: American Scientific Publishers; 2004. pp. 627-38. 